\title{
Estudo de cortadoras a laser open source e seus problemas mais comuns
}

\author{
Breno Lear Martins Guethi
}

\begin{abstract}
Resumo - O advento de metodologias, tal qual a do Design Thinking, que incentivam a prototipagem rápida como forma de verificação e validação de ideias e produtos levou à um aumento da procura por ferramentas que permitiam essas práticas - Impressora 3D, placa Arduíno e Protoboard, Máquina de Corte à Laser. Muitos desses mecanismos e softwares têm vertentes centradas no open source - modelo que deixa o programa e os métodos de montagem abertos a todos os interessados. São muito utilizados em laboratórios de prototipagem rápida, de ensino e em FabLab's. Para aumentar ainda mais a acessibilidade da cortadora à laser, esse trabalho tem por objetivo estudar os conceitos, softwares e hardwares acerca dessa máquina pautando-se em três modelos existentes e abertos, apresentar sugestões acerca de sua montagem e soluções de problemas recorrentes.
\end{abstract}

Palavras-chave - Prototipagem rápida; Máquina de corte a laser; Open source; Cortadora a laser

\section{Introdução}

Com o advento de metodologias pautadas na prototipagem rápida, tais quais o Design Thinking, máquinas que auxiliam esse processo passaram a ser mais visadas. Dentre elas, destacam-se a Impressora 3D (tridimensional por deposição de material), a placa Arduíno, a cortadora a laser. A importância desses produtos é tal que não é necessário fazer uma manufatura, usinagem, projeto técnico de uma ideia. Aproxima-se quem tem a concepção do modelo físico e facilita-se o ciclo do design thinking - descobrir, definir, desenvolver, redefinir, refinar, entregar (BROWN, 2010).

Sabe-se que por meio do trabalho de ilustres estudiosos da Física, dentre eles Albert Einstein, Max Planck, Niels Bohr, Erwin Schrödinger, Werner Heisenberg, foram analisadas as formas da matéria e suas variações. A energia, a luz, as partículas, os elétrons a foram decisivas para Gordon Gould que, em 1959, descreveu o laser em "The LASER, Light Amplification by Stimulated Emission of Radiation" (GOULD, 1959) e para Theodore Harold Maiman que, em 1960 pôde produzir o primeiro do tipo

Foi com o aprimoramento dessa tecnologia, junto do uso de associação de lentes, que o Laser para manufatura foi viável.

A primeira máquina de corte à laser foi feita pela Western Electric Research Engineering Center - em 1965- e era, inicialmente, utilizada em indústrias de fabricação para cortar materiais e ferramentas com geometrias complexas. Aprimorada pelo Reino Unido, que em 1967 passou a utilizar os lasers de oxigênio para cortar metais, essa tecnologia foi, posteriormente, colocada em produção a fim de cortar titânio para aplicações aeroespaciais - em 1970. Isso ocorria concomitantemente ao aprimoramento do corte com laser de Dióxido de Carbono (CO2) utilizado, até o momento, em materiais não-metálicos, uma vez que ainda não tinha potência suficiente para transpassar metais.

O corte desse tipo de máquina ocorre por meio de alguns fenômenos: corte de vaporização, derreter e fundir, rachaduras por estresse térmico, corte reativo. $\mathrm{O}$ mais comum para os materiais usuais é o corte de vaporização. Neste processo o que ocorre é que o feixe do laser focado na superfície do material o faz chegar em seu ponto de sublimação gerando um furo que aumenta a absortividade pelo material aumentando e aprofundando o furo.

Atualmente, por conta da inauguração de diversos laboratórios de fabricação (FabLab's que disponibilizam gratuitamente máquinas e materiais para prototipagem rápida, cursos, aulas,

Versão inicial submetida em 20 de julho de 2017. Versão final aceita em 21 de setembro de 2017. Publicado em 16 de novembro de 2017. Digital Object Identifier 10.11606/issn.2526-8260.mecatrone.2017.134667 
assistência especializada) no mundo, houve uma difusão maior das tecnologias de prototipagem, o que estimulou não só a indústria e as instituições de ensino, mas também o grande público. Esse interesse aumentou a busca pelas tecnologias fornecidas, aprimoramentos, customizações, reduções nos preços, maior facilidade de encontrar certo modelo no mercado - para compra ou para construção.

No que diz respeito à construção, ainda não se está em um ponto ótimo do produto, já que por terem desenvolvimento aberto, muitas vezes, não apresentam praticidade, robustez, facilidade de construção para quem não está ativamente envolvido ou não tem conhecimento eletromecânico. Também, muitas vezes apresentam fios expostos, placas sem acabamento, sem proteção, por serem considerados supérfluos.

Outro fato que dificulta a otimização da cortadora à laser open source é o custo elevado de um laser de alta potência - necessário para cortar chapas de madeira, acrílico, papel grosso. De certa forma, esse é um limitante complicado no projeto, já que as lentes e o laser requeridos, tem alto valor agregado, são difíceis de produzir.

Dois dos protótipos estudados aqui (Instructables e Fun of DIY) utilizam lasers de baixa potência enquanto que o da Lasersaur faz uso de um canhão laser de alta potência com lentes focalizadoras.

\section{Laser}

Os lasers que estão presentes no mercado atual são dos mais diversos. Não somente em termos de espectro e potência, mas também pelo aumento da difusão para a locais que permitem à população usufruir - lasers de baixa potência para aplicações sensoriais em brinquedos, de alta potência em cortadoras lasers profissionais presentes nos FabLab's. Algumas potências de laser podem ser vistas na Tabela I.

Tabela I - Aplicações e potências lasers usuais

\begin{tabular}{|l|c|}
\hline \multicolumn{1}{|c|}{ Aplicação do Laser } & Potência $(\mathbf{m W})$ \\
\hline Pointer - apontador & 1 a 5 \\
\hline DVD e CD player & 5 a 10 \\
\hline Gravador de CD e de DVD & 100 a 250 \\
\hline Laser verde - disco holográfico & $1 \mathrm{k}$ \\
\hline Lasers de micro usinagem & $1 \mathrm{k} \mathrm{a} 20 \mathrm{k}$ \\
\hline Laser CO2 para corte & $30 \mathrm{k} \mathrm{a} 100 \mathrm{k}$ \\
\hline Laser CO2 para corte industrial & $100 \mathrm{k}$ a 3000k \\
\hline
\end{tabular}

As potências 30k 100k também são usadas para aplicações cirúrgicas.

\section{Instructables}

Idealizado pelo site conhecido por modelos e projetos de eletrônica com Arduíno, essa versão de cortadora é pequena e funciona mais como um gravador, já que se utiliza de um laser de baixa intensidade. O tutorial de montagem apresentado no site explica que alguns componentes são comprados prontos, ou desmontados de sucatas. A montagem pronta é mostrada na Figura 1.

Os materiais necessários para a montagem são poucos, sendo que alguns dos componentes usados, como o driver de DVD, podem conter mais de uma peça importante, mostrado na Tabela II.

Nesse modelo, serão utilizados os motores de passo e os lasers dos drivers do DVD para

fazer a plataforma móvel do laser e da base. Com isso será possível ter movimentos nos eixos x e y. Monta-se o laser a partir do driver do DVD dentro de um housing. 
Figura 1 - Máquina de corte a laser open source.

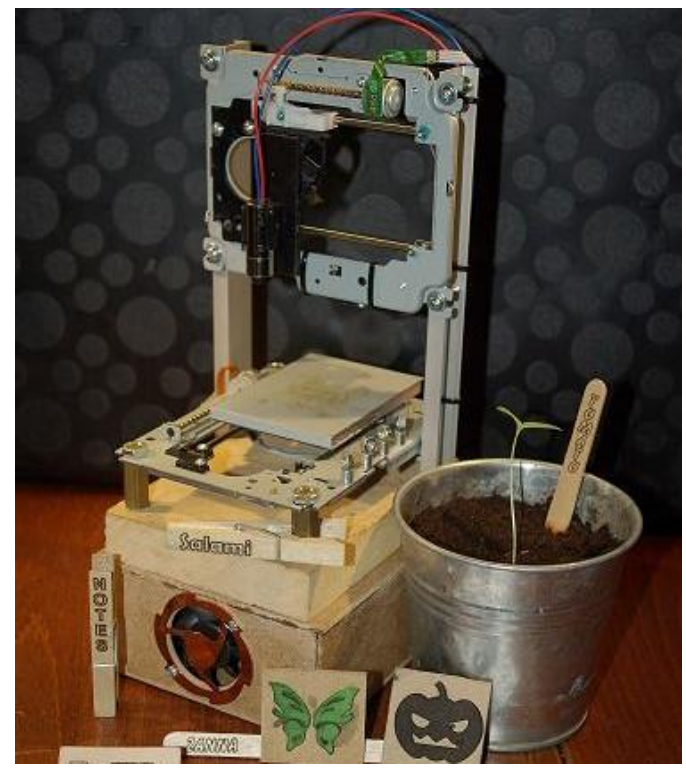

Fonte: Instructables.

Tabela II - Lista de materiais cortadora a laser open source. Fonte: Instructables.

\begin{tabular}{|l|c|}
\hline \multicolumn{1}{|c|}{ Material } & Número de componentes \\
\hline Arduíno - controlador & 1 \\
\hline Easydrive - driver de passo & 2 \\
\hline Driver de DVD - motor de passo e diodo laser & 2 \\
\hline Laser housing - invólucro para o emissor & 1 \\
\hline Laser driver - LM317 - feedback positivo & 1 \\
\hline Outros - porcas, parafusos, materiais estruturais & - \\
\hline
\end{tabular}

Outros materiais são diversos, e não são elencados quantos devem ser utilizados

Para a parte estrutural, a montagem é descrita de forma tal que o laser é posicionado acima da base onde será o corte, distando 7,5 polegadas (19 centímetros) por pequenas estruturas de perfil $\mathrm{C}$ mostradas na Figura 2. Sua montagem utiliza um em cada lateral da base, mostrado na Figura 3.

A parte eletrônica descrita é montada de forma a ficar fora da estrutura ativa. Nela ficarão o Arduíno, os dois Easydriver, e um opcional rele que ligaria um ventilador. A montagem básica pode ser vista na Figura 4.

Figura 2 - Viga de sustentação de perfil C.

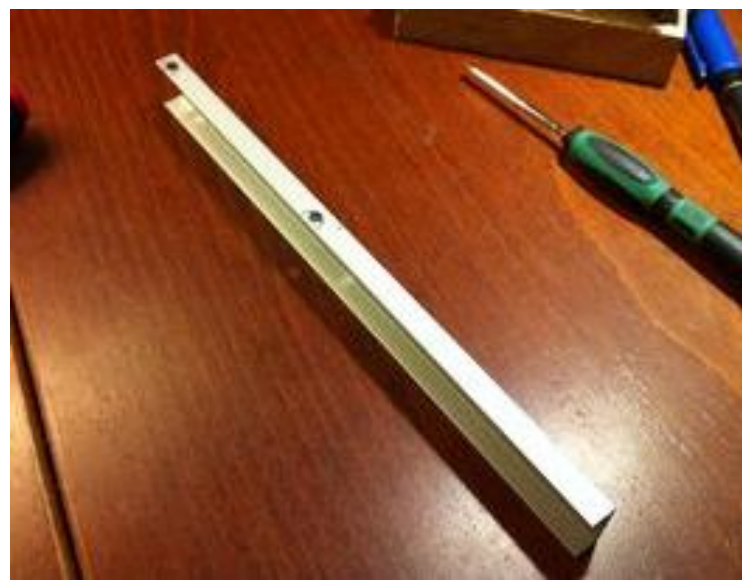

Fonte: Instructables. 
Figura 3 - Encaixe vigas com base e estrutura superior do laser.

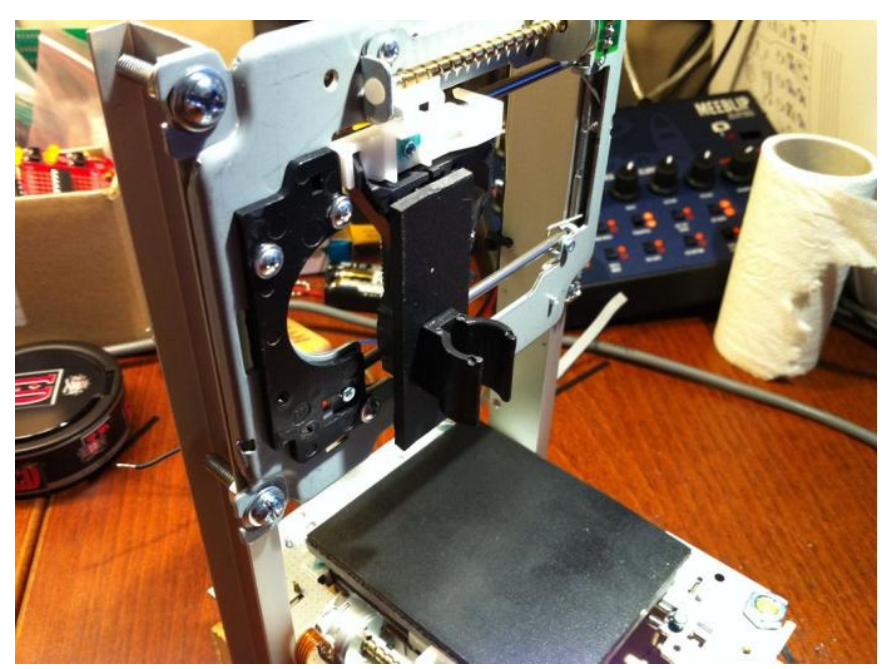

Fonte: Instructables.

Figura 4 - Montagem eletrônica básica.

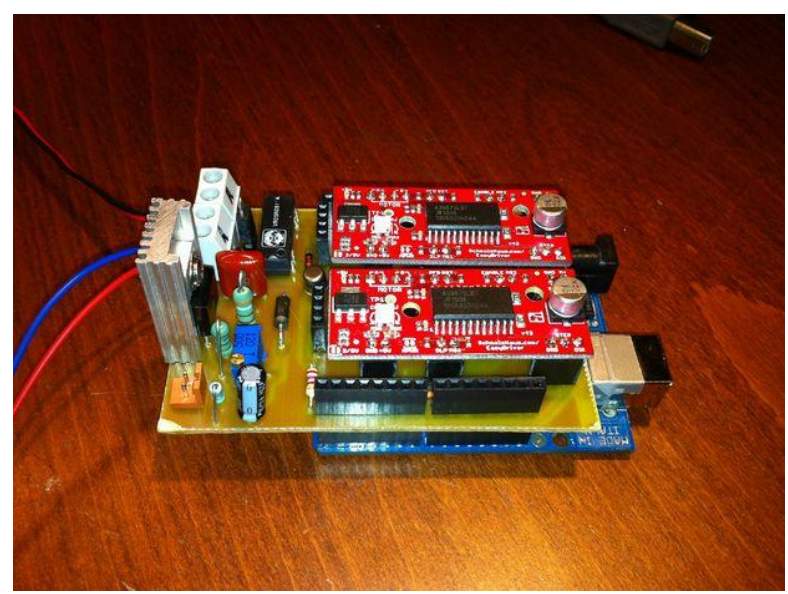

Fonte: Instructables.

O programa de computador indicado para realizar o desenho a ser cortado é o Inkscape software aberto para edição de vetores. Junto dele, deve ser instalada uma extensão chamada Laser engraver, que gerará o $G$-code do CNC. Por fim, deve-se baixar o programa $G$-code sender, que se comunicará via GRBL - software que interpreta o G-code.

\section{Lasersaur}

O tutorial apresentado pela Lasersaur é o mais completo, porém o mais complexo dos três modelos encontrados. Basicamente, é ensinado como fazer uma cortadora à laser que beira o profissional, em tamanho e complexidade. O modelo montado é mostrado na Figura 5.

O site propõe uma lista de peças já orçadas para a Europa e para os Estados Unidos da América. $\mathrm{O}$ esquema de montagem é dado por um modelo 3D fornecido na Figura 6, que visa auxiliar durante a montagem. É válido notar que aqui a cortadora já é fechada o que não acarretará em problemas com gases tóxicos da queima que ocorre. Há um sistema de escape desses gases feito por um exaustor/ventoinha.

As etapas de construção são descritas no site, tendo o passo a passo dos elementos estruturais, dos carros de movimento x e y, do laser. A parte eletrônica, esquema dos fios tem uma seção dedicada, com intuito de ensinar, minimamente, o que precisa ser feito para que o sistema funcione. 
Figura 5 - Cortadora a laser.

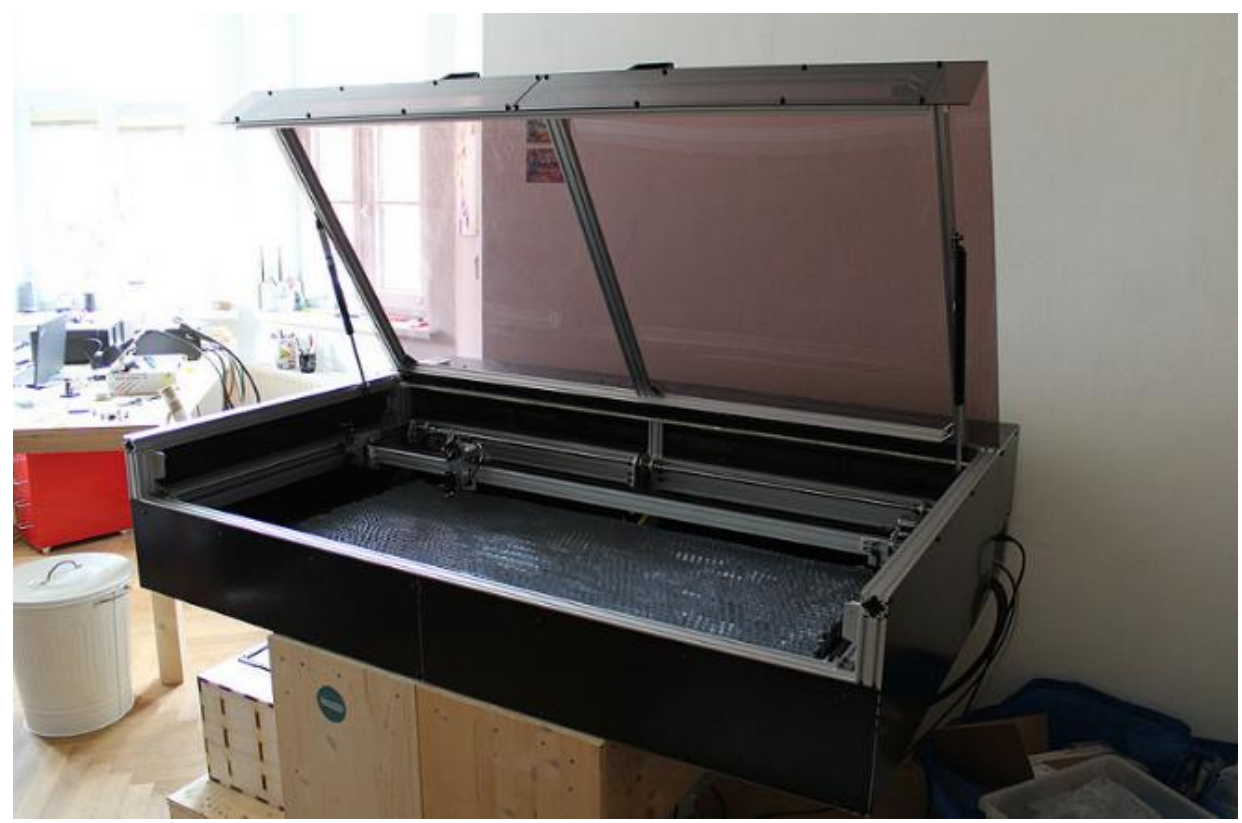

Fonte: Lasersaur.

Figura 6 - Esquema da montagem da cortadora.
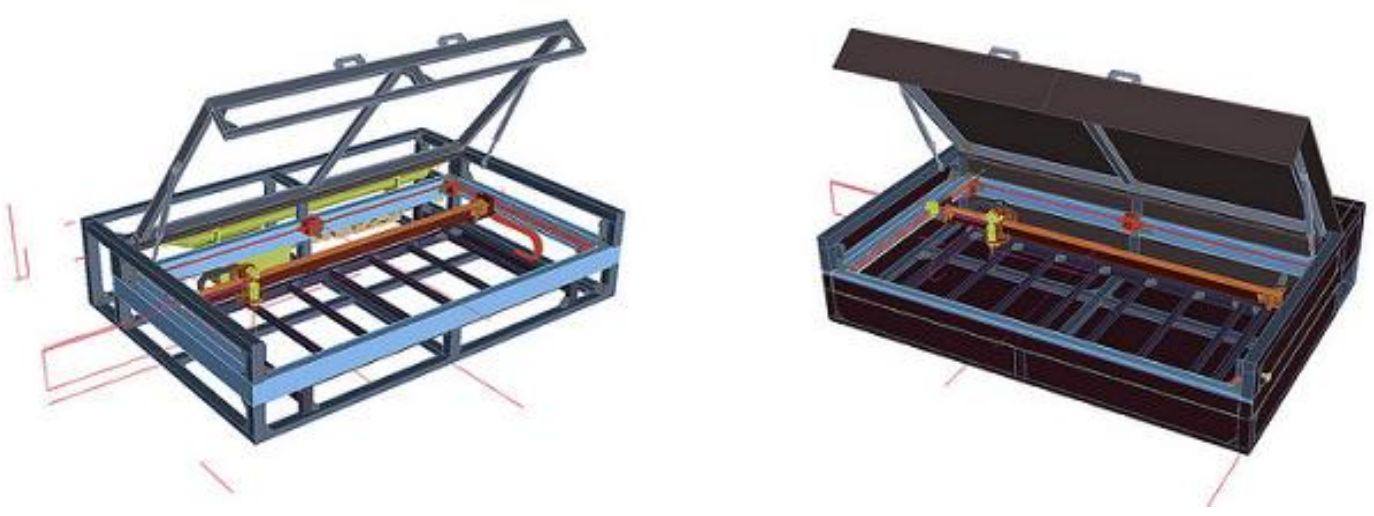

Fonte: Lasersaur.

Em termos de software, a franquia disponibiliza o programa próprio aberto para quem desejar utilizar e modificar. Ele funciona como mediador entre o arquivo vetor (o desenho) e a cortadora - tanto a intensidade do laser definida por padrões de cores pré-selecionados, quanto o movimento do carro.

\section{Fun of DIY}

O blog de eletrônica Fun of DIY, apresentado nas referências, criou um modelo a partir das cortadoras vistas nos fóruns. Também se faz o uso de laser de baixa intensidade, já que é mais de fácil acesso. Incentiva-se o reuso de componentes, contudo, esse protótipo aparenta ser mais avançado em termos de refinamento e acabamento, visto na Figura 7.

Os materiais necessários para a montagem, bem como os do modelo da Instructables, são, em sua maioria, reutilização de componentes, tais quais drivers de DVD's. Uma diferença para o primeiro modelo apresentado, é a utilização de um microprocessador - a Raspberry P $i$ - diferindo do controlador Arduíno. Os materiais necessários para a montagem são descritos pela Tabela III.

Assim como no modelo estruturado pelo Instructables, esse utiliza os motores de passo e os lasers dos drivers do DVD para fazer a plataforma móvel do laser e da base, com intuito de fornecer movimento nos eixos x e y, e para laser junto do invólucro. 
Figura 7 - Máquina de corte a laser.

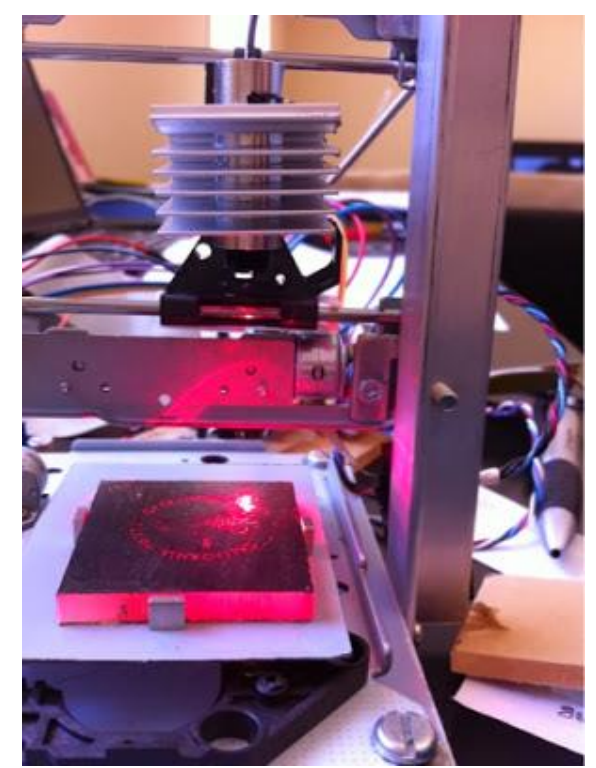

Fonte: Fun of DIY

Tabela III - Lista de materiais. Fonte: Fun of DIY

\begin{tabular}{|l|c|}
\hline \multicolumn{1}{|c|}{ Material } & Número de componentes \\
\hline Raspberry $\mathrm{P} i$ - controlador & 1 \\
\hline Ponte H - duas para cada motor de passo & 4 \\
\hline Driver de DVD - motor de passo e diodo laser & 2 \\
\hline Laser housing - invólucro para o emissor & 1 \\
\hline Laser driver - LM317 - feedback positivo & 1 \\
\hline Outros - porcas, parafusos, materiais estruturais & - \\
\hline
\end{tabular}

Outros materiais são diversos, e não são elencados quantos devem ser utilizados

É ensinado como montar o laser a partir do diodo retirado do DVD - dentro da carcaça dele há dois, um infravermelho, que não será utilizado, e um vermelho de 650nm. Nesse último, deve-se descobrir o que é cada uma das entradas - NC, cátodo e ânodo - e verificar se a resistência não está muito alta. Caso esteja, não poderá ser utilizado. Deverá, então, ser montado dentro do invólucro e conectado aos fios que controlarão o modelo.

Para a parte estrutural, a montagem é feita de forma igual a do primeiro modelo. O laser é posicionado acima da base onde será o corte, distando 7,5 polegadas (19 centímetros) por pequenas estruturas de metal, uma em cada lateral da base.

Os programas recomendados para a geração da imagem, bem como na primeira seção, são o Inkscape, Inkscape-Laser-engraver-Extension. Eles gerarão um código em G que deverá ser passado para RPI, e mover para a pasta onde terá os outros códigos que controlam a cortadora.

\section{Sugestões}

Dos projetos abertos verificados, há diversas diferenças que valem ser citadas. A primeira delas é o tamanho díspar da Laserssaur em relação às outras - o que não é necessariamente negativo, já que os modelos menores têm área útil pequena. Caso haja uma monetização do projeto, seria mais atrativo uma área de corte maior.

Deve-se pensar no momento de fazer um modelo que existem melhorias que devem ser implementadas para um uso sem preocupações - seja pelos gases de escape, seja pelo contato humano no laser. Para isso, sugere-se uma estrutura externa na qual seriam acopladas placas que poderão fechar a estrutura para que não haja contato com a máquina em operação. Além disso, pode 
ser feita, facilmente, uma porta para que se tenha acesso à plataforma de corte, bem como uma saída de ar, com um pequeno exaustor, ou uma ventoinha de computador.

\section{Problemas levantados na comunidade Open Source}

Os problemas mais comuns levantados para uma cortadora à laser são os travamentos ocasionais que ocorrem durante uma impressão, o erro do 'zero' mudar de referência e falhas quando o laser é requerido em plena potência (em 100\%). Verificaram-se os motivos desses erros e formas de previní-los.

\subsection{Travamento}

Caracterizado pela guinada do laser no meio da impressão que ocasiona diversas imprecisões no corte. Seus motivos principais são dois: baixo refinamento do software utilizado (ao tentar interpretar uma forma que não conseguiu discretizar por completo, interpreta de maneira errônea e leva o mecanismo a realizar um movimento errado) ou baixa qualidade dos motores utilizados.

Quando os motores são de baixa qualidade, o problema é mais facilmente resolvido, uma vez que basta trocar os motores obsoletos, usados, por um modelo mais preciso. Este é o caso mais usual nas comunidades online, uma vez que, reutiliza-se muitos materiais, o que não é recomendado.

\section{2 'Zero' referenciado}

Outro problema comum ocorre quando o que é cortado tem proporções grandes. Nele a máquina não retorna para a posição origem inicial definida no programa, o que pode comprometer o corte que atual e se houver uma sequência requerida, outros.

Pelas respostas encontradas sua principal causa é o uso de placas de baixa qualidade e confiabilidade - hobbyCNC é a que mais causa problemas.

\subsection{Laser em plena potência}

Os problemas que ocorrem quando o laser é requisitado $100 \%$ são algumas falhas no corte devido ao alto nível de solicitação. Pode ser visto na Figura 8 um exemplo de corte com falhas por esse tipo.

Figura 8 - Placa em MDF com falhas de continuidade.

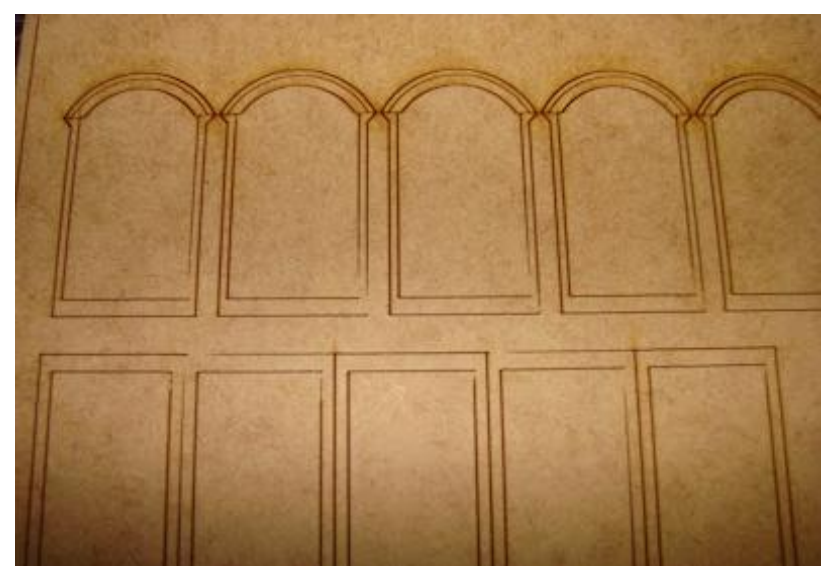

Fonte: Lapac.

Recomendam-se duas soluções para isso: diminuir a potência e a velocidade utilizada, concomitantemente, ao aumento do número de vezes de passagem do laser no segmento de linha; alterar no modelo do computador criando linhas que sobreporão as reais distando metade da resolução da cortadora, como visto na Figura 9. 
Figure 9 - Modelo CAD com sobreposição de linhas.

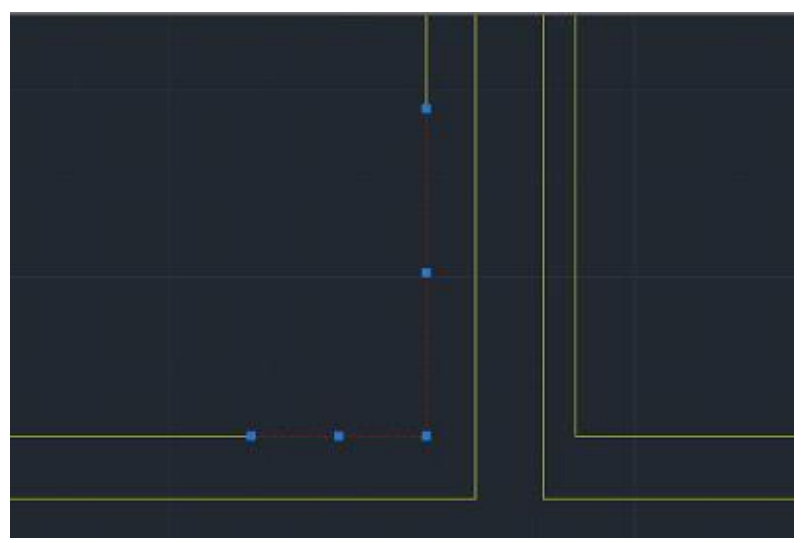

Fonte: Lapac.

\section{AGRADECIMENTOS}

Primeiramente, a Deus por me dar vida, entendimento, força, saúde.

Aos meus pais, Duvivier e Gislaine, irmão, Miguel, e minha namorada, Barbara, pelo amor, incentivo, paciência e apoio incondicional.

À Escola Politécnica da Universidade de São Paulo, pela oportunidade de fazer meu curso.

Ao Professor Doutor Celso Massatoshi Furukawa pelo suporte no pouco tempo que lhe coube, pelas suas correções e incentivos.

\section{REFERÊNCIAS}

Azevedo, Fábio Mariotto de, Estudo e projeto de melhoria em máquina de impressão 3D.

Graduado. São Paulo, Tese, Escola de Engenharia de São Carlos da Universidade de São Paulo, São Carlos, 2013.

Brown, T. Design Thinking - uma metodologia poderosa para decretar o fim das velhas ideias. Rio de Janeiro, Elsevier, 2010.

Fun of DIY, A Raspberry Pi controlled mini CNC Laser engraver. Disponível em:

<http://funofdiy.blogspot.com.br/2013/10/a-raspberry-pi-controlled-mini-laser.html>. Acesso em: 02 de outubro de 2016.

Garcia, Luis Hilário Tobler, Desenvolvimento e fabricação de uma mini-impressora 3D para cerâmicas. Mestre. Orientador Benedito de Moraes Purquerio, Escola de Engenharia de São Carlos da Universidade de São Paulo, São Carlos, 2010.

Gould, G. The Laser, Light Amplification by Stimulated Emission of Radiation. The Ann Arbor Conference on Optical Pumping, Universidade de Michigan. Anais. Franken: 1959. OCLC 02460155 .

\section{Industrial. 2500mW Desktop DIY Violet Laser Engraving Kits Engraver CNC Printer} Assembling | eBay. Disponível em: <http://www.ebay.com/itm/2500mW-Desktop-DIY-VioletLaser-Engraving-Kits-Engraver-CNC-Printer-Assembling-/291921067539>. Acesso em: 6 nov. 2016.

Instructables, Pocket laser engraver. Disponível em: <http://www.instructables.com/id/Pocketlaser-engraver/>. Acesso em: 3 de outubro de 2016. 
Lapac, Dicas Rápidas para Laser Cutter: Laser "falhando". Disponível em:

<http://diariodolapac.blogspot.com.br/2013/04/da-serie-dicas-rapidas-para-laser.html>. Acesso em: 8 nov. 2016.

Martin, D. Theodore Maiman; Demonstrated First Laser. Disponível em:

<http://www.nytimes.com/2007/05/11/obituaries/11maiman.html?_r=0>. Acesso em: 01 de outubro de 2016.

Manual Nortd Lasersaur. Lasersaur. Disponível em: <https://github.com/nortd/lasersaur/wiki>. Acesso em: 01 de outubro de 2016.

Weber, Marvin J. Handbook of laser wavelengths - Ed. Marvin J. Weber, Boca Raton: CRC Press LLC, 1999.

\title{
Title - Study of open source laser cutters and their common problems
}

\begin{abstract}
The advent of methodologies, such as Design Thinking, which encourage rapid prototyping as a way to verify and validate ideas and products, led to an increase in the demand for tools that allowed these practices - 3D Printer, Arduíno and Protoboard, Laser cutting machine. Many of these mechanisms and softwares have a focus on open source - a model that leaves the program and assembly methods open to all concerned. They are widely used in fast prototyping, teaching and FabLab's laboratories. To further increase the accessibility of the laser cutter, this work aims to study the concepts, software and hardware about this machine based on three existing and open models, present suggestions about its assembly and solutions to recurring problems.
\end{abstract}

Keywords - Rapid prototyping; Laser cutting machine; Open source; Laser cutter;

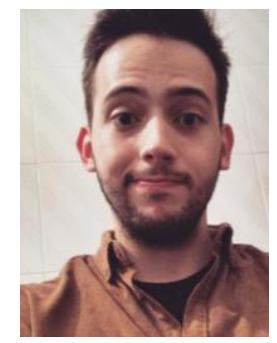

Breno Lear Martins Guethi, Aluno do $5^{\circ}$ ano do curso de Engenharia Mecânica, Escola Politécnica da USP 